\title{
On the formation of expanding crack tip precipitates
}

\author{
Wureguli Reheman • Per Ståhle • Martin Fisk • \\ Ram Niwas Singh
}

Received: 14 November 2018 / Accepted: 17 April 2019 / Published online: 2 May 2019

(C) The Author(s) 2019

\begin{abstract}
The stress driven growth of an expanding precipitate at a crack tip is studied. The material is assumed to be linearly elastic, and the expansion is considered to be isotropic or transversely isotropic. The extent of the precipitate is expected to be small as compared with the crack length and distance to boundaries. The problem has only a single length scale given by the squared ratio of the stress intensity factor and a critical hydrostatic stress that initiates the growth of the precipitate. Therefore, the growth occurs under selfsimilar conditions. The equations on non-dimensional form show that the free parameters are expansion strain, degree of anisotropy and Poisson's ratio. It is found that the precipitate, once initiated, grows without remote load for expansion strains above a critical value. The anisotropy of the expansion strongly affects the shape
\end{abstract}

\footnotetext{
W. Reheman ( $\square)$

Department of Mechanical Engineering, Blekinge Institute of Technology, Karlskrona, Sweden e-mail: wureguli.reheman@bth.se

P. Ståhle

Solid Mechanics, Lund University, Lund, Sweden e-mail: per.stahle@solid.lth.se

\section{Fisk}

Materials Science and Applied Mathematics, Malmö

University, Malmö, Sweden

e-mail: martin.fisk@mau.se

\section{R. N. Sing}

Mechanical Metallurgy Division, Bhabha Atomic Research Centre, Mumbai 400085, India

e-mail: rnsingh@barc.gov.in
}

of the precipitate but does not have a large effect on the crack tip shielding.

Keywords Growth of precipitates - Stress driven · Anisotropic expansion - Crack tip shielding . Zirconium hydride

\section{Introduction}

Hydrogen embrittlement, or hydrogen degradation, is the loss of mechanical strength of metals and alloys caused by the interaction with hydrogen. The embrittlement is often manifested as the loss of ductility and fracture toughness, or a change of failure mode, see Puls (2012) and Louthan Jr (2008). To explain the hydrogen embrittlement, a number of hydrogen related failure mechanisms have been proposed during the years. Hydride induced embrittlement, hydrogen enhanced localised plasticity, hydrogen enhanced decohesion, and grain boundary mechanisms are a few examples. In these typical cases, the hydrogen directly affects the material's load bearing capability. For other materials, such as zirconium, titanium, niobium, vanadium, a second phase is formed when the metal is exposed to hydrogen under specific conditions, eg. in Garverick (1994). The process when the hydride, which is a brittle phase, forms and fails at a load level that is much lower than the original material is called hydride embrittlement, see Courtney (2005). The most common form of hydride embrittlement is delayed hydride crack- 
ing. Delayed hydride cracking is a manifestation of a localised form of hydride embrittlement. It is caused by hydrogen migration along hydrostatic stress gradients and forms a brittle phase. The crack that migrates through the brittle phase stops when the tip reaches to the matrix material, and the formation of hydride and developing of crack through the hydride repeats itself until the crack reaches its critical size and the material fails, cf. Singh et al. (2002) and Louthan Jr (2008). This is a typical problem in nuclear power plants, where zirconium is extensively used for encapsulation of fuel and control rods because of its low neutron absorption cross section, high strength and good corrosion resistance (Moan and Rudling 2002).

The formation of hydrides is known to occur when the hydrogen concentration exceeds a critical level. Typical places where hydrides may form are at notches, crack tips, dislocations, etc., since hydrogen is transported along the positive gradients of hydrostatic stresses and is known to accumulate at a place where the hydrostatic stress has a local maximum. Several experimental and theoretical studies by Bertolino et al. (2003), Cann and Sexton (1980), Ellyin and Wu (1994) and Farrow and Watkins (1965) have focused on the hydride formation at crack tips, and its effect on strength. Models of crack propagation based on diffusion controlled mechanisms have been studied by Svoboda and Fischer (2012) and Shi (1999). The terminal solid solubility of hydrogen in $\mathrm{Zr}-2.5 \mathrm{Nb}$ alloy has been studied by Singh et al. (2005). Temperature gradients and hydrogen contents related models have been investigated by Turnbull et al. (1996)

In a material exposed to hydrostatic stress, an expanding volume leads to a release of free energy. As a consequence, the hydrostatic stress thereby exerts a driving force that stimulates metal to hydride transition. During the transition process the material undergoes volumetric changes that often are very large, see Carpenter (1973) and Weatherly (1981). The stress distribution and the mechanical conditions surrounding the crack tip will be affected by this and hence the risk of crack growth. However, there are very few studies on stress driven formation of precipitates where the effect of the volumetric changes are considered in the calculation of the crack tip stress distribution by Lufrano and Sofronis (1996).

In this work, we study the formation of expanding precipitates near a crack tip by considering a volumetric expansion during the precipitation. The growth of the precipitates are assisted by a large hydrostatic stress that drags hydrogen atoms to the crack tip region, at a critical hydrogen concentration a chemical reaction initiates the precipitation. The transformation introduces an expansion of the material. Both isotropic and anisotropic expansion are investigated. The anisotropic expansion is limited to transversely isotropic expansion. Later in this text, the anisotropic expansions refer to transversely isotropic case. The material is assumed to be linear elastic and the precipitate is assumed to be small compared to the crack length, the distance from crack tip to the closest boundary, and other characteristics of the geometry. It is further assumed that the diffusion process is sufficiently fast as compared to the growth of precipitates so that the hydrogen distribution can be considered to be in a equilibrium everywhere.

The ductile properties of metal matrices are usually lost when the precipitate is formed. With loss of ductility the stress at failure increases and in an elastic crack tip stress field the region of non-linear stress decrease. The size of the region scales quadratically with the failure stress which quite commonly means that the nonlinear region may be treated as embedded in a square root singular stress. Loss of ductility is an observed property of many precipitates such as hydrides, oxides, general ceramic materials and similar. In the precipitates considered here the material is more likely to loose strength through cleavage. The fracture processes including other non-linear material behaviour is assumed to be confined to a very small region that in the model is treated as a point. The choice we did reduces the number of materials that can be considered but also selecting a specific yield stress would do the same and in addition increase the number of free parameters. Should the yield stress be very low the free elastic energy may be reduced significantly, which would impede the growth of the precipitate. In this study, it is assumed that the yield stress is sufficiently high as compared with critical stress for formation of precipitates.

A non-dimensional formulation of the problem reveals that only three parameters are present, i.e. the expansion strain, the degree of anisotropy, and Poisson's ratio. Here, Poisson's ratio is not varied but the other two free parameters are varied. A single length scale implies that the precipitate must grow under selfsimilar conditions. It is also readily observed that there is a critical expansion strain above which the precipitate grows without remote load once it is initiated. Under 
that critical load, the precipitate size is controlled by the remote stress intensity factor. A finite element (FE) model is used to obtain details regarding precipitate shapes, size and crack tip stress intensity factors. The problem is analysed as a boundary layer problem for a given remote square root singular stress field. The results show that the crack tip becomes partly embedded in the precipitate.

The material model including the volumetric expansion and the boundary value problem is defined in Sect. 2. The non-dimensional form of the field equations show that the model only contains two free parameters. In Sect. 3 the solution procedure using the FE method is described. The results of the FE calculations in terms of the effect of the precipitate on the stress field and the evolution of the precipitate shape accompanied by a discussion are presented in Sect. 4 . Finally a derivation of the precipitate size and the critical expansion leading to a spontaneously self-growing precipitate is derived in the "Appendix".

\section{Model}

A model for stress induced precipitate formation at a crack tip is described. The crack tip is assumed to be sharp and the fracture processes are confined to a point. The formation process is supported by constituent (in the form of ions, atoms, molecules or substances) that are driven through the material by the concentration gradient, as well as by the hydrostatic stress gradient. The motivation for using a stress criteria for the precipitation is explained. The material deformation coupled with precipitate expansion is defined. At the end of the section, the governing equations are transferred into a non-dimensional form.

\subsection{Criteria for formation of precipitate}

In general, constituent will diffuse along gradients of stress, chemical concentration, temperature, electric potential etc. In this study, it is assumed that precipitate formation proceeds in a quasi-static manner under constant temperature, potential etc. Also for simplicity, the source of constituent is assumed to be inexhaustible and present in a solid solute form within the material matrix. The transport of ions/atoms is supposed to be driven both by the gradient of the concentration and by the gradient of the hydrostatic stress. Therefore, the Einstein-Smoluchowski equation (cf. Li 1978) for stress driven flux, $J_{i}$, is used as follows,

$J_{i}=-D C_{, i}+\frac{D C V}{R T} \sigma_{h, i}$,

where $D$ is the diffusivity constant, $C$ is the concentration, $V$ is the partial molar volume, $R$ is the universal gas constant, $T$ is the absolute temperature, and $\sigma_{h}$ is the hydrostatic stress. The writing ()$_{, i}$ denotes the partial derivative with respect to the spatial Cartesian coordinate $x_{i}$. The subscripts $i, j, k$ assume values 1 , 2 or 3 and on these Einstein's summation rule is used. The hydrostatic stress $\sigma_{h}$ is given by

$\sigma_{h}=\frac{1}{3} \sigma_{k k}$,

where $\sigma_{k k}$ is the trace of the stress tensor, $\sigma_{i j}$.

In this work, it is assumed that the mechanical state changes slowly as compared with the change of the chemical state and the rate of formation of the precipitate as the remote load considered to be quasi-static or practically constant. Thus, it is suggested that the total flux of ions $J$ is small, so it may be considered to be negligible. Thereby, Eq. (1) is reduced to

$-C_{, i}+\frac{C V}{R T} \sigma_{h, i}=0$.

After integrating the left hand side terms of Eq. (3), one obtains

$\sigma_{h}=\sigma_{o}+\frac{R T}{V} \ln \left(\frac{C}{C_{o}}\right)$,

where $\sigma_{o}$ is the remote stress, and $C_{o}$ is the ambient concentration at large distance away from the crack tip. At equilibrium in the absence of mechanical load, the ambient concentration would be the concentration in the entire body.

As stated previously, the material is assumed to form a precipitate when the concentration reaches a critical level, say $C_{c}$ (Singh et al. 2005). As the remote stress $\sigma_{o}$ at large distances from the crack tip is considered to be vanishingly small or insignificant as compared with the square root singular stresses in the crack tip region, thus the stress $\sigma_{o}$ can be ignored and the corresponding critical hydrostatic stress $\sigma_{c}$ is obtained as

$\sigma_{c}=\frac{R T}{V} \ln \left(\frac{C_{c}}{C_{o}}\right)$.

Thus, a precipitate is formed when the hydrostatic stress reaches the critical stress, $\sigma_{h}=\sigma_{c}$, in the material. This means that first the stress field only influenced 
by remote stress, however once the precipitates start to form the stress field also influenced by the formation of hydride. The transformation of precipitates in this study is assumed to be irreversible.

If the matrix material expands already before precipitations form due to the presence of the constituent in solid solution, this may be considered. Therefore, if the dilatational free hydrostatic stress is proportional to the concentration $C$, this would give $\sigma_{h}=\sigma_{h}^{e}+\sigma_{h}^{s}=$ $\sigma_{h}^{e}+\alpha C$, where $\alpha$ is a material parameter. It is readily seen that the critical stress $\sigma_{c}^{\prime}$ in this case becomes $\sigma_{c}+\alpha\left(C_{c}-C_{o}\right)$, where $\sigma_{c}$ is the critical according to Eq. (5). For more complicated dependencies integration may have to be performed numerically. Never the less the critical concentration criterium may be replaced with a critical stress.

\subsection{Material model}

The analysis concerns the mechanical state of the crack tip neighbourhood as exposed to remote loads and constraints during expansion of a precipitate, e.g., during the phase transformation from matrix to precipitate. The total strains $\varepsilon_{i j}$ are assumed to be small and are defined as gradients of displacements $u_{i}$ as

$\varepsilon_{i j}=\frac{1}{2}\left(u_{i, j}+u_{j, i}\right)$,

The total strains are decomposed into an elastic part $\varepsilon_{i j}^{e}$, and an expansion part $\varepsilon_{i j}^{s}$ as follows

$\varepsilon_{i j}=\varepsilon_{i j}^{e}+\varepsilon_{i j}^{s}$,

where the elastic strain is defined by Hooke's law

$\varepsilon_{i j}^{e}=\frac{1}{E}\left[(1+v) \sigma_{i j}-v \delta_{i j} \sigma_{k k}\right]$.

The $v, E$ and $\delta_{i j}$ are Poisson's ratio, the modulus of elasticity and Kronecker's delta, respectively. Furthermore, the expansion strain is defined as

$\varepsilon_{i j}^{s}=\Lambda\left(\delta_{i 1} \delta_{j 1} e_{1}+\delta_{i 2} \delta_{j 2} e_{2}+\delta_{i 3} \delta_{j 3} e_{3}\right)$,

where $e_{i}$ are the principal expansion strains and in directions coinciding with the $x_{i}$ axes. The parameter $\Lambda$ is one in the precipitate and zero in the original material according to

$\Lambda= \begin{cases}1 & \text { if }\left(\sigma_{h}\right)_{\max } \geq \sigma_{c} \\ 0 & \text { if }\left(\sigma_{h}\right)_{\max }<\sigma_{c} .\end{cases}$
The stress $\left(\sigma_{h}\right)_{\max }$ is the largest hydrostatic stress until present time. The system is not rate dependent and time is used to compile with the incremental nature of the problem. The critical stress $\sigma_{c}$ is given by Eq. (5).

According to the choice made in Eq. (10)

the expansion is either on or off depending on the material phase. In general constituents in solid solution expand or occasionally decrease the volume occupied by the matrix material. In the present study it is assumed that the constituent in solid solution does not cause a significant expansion or due to constraint does not cause an additional stress. An example of such a material system is zirconium and hydrogen together forming zirconium hydride. According to Puls (2012) and Louthan Jr (2008) the effect of hydrogen in solid solution is small due to the depletion of hydrogen in a large region surrounding the hydride, due to so called uphill diffusion close to the precipitate.

The expansion of the precipitate is supposed to be isotropic or transversely isotropic with a basal plane, in which the expansion is isotropic. The basal plane is assumed to coincide with the crack plane. It is assumed that the transversely isotropic expansion has the following principal strains

$e_{2}=(1+2 q) \varepsilon_{s}$ and $e_{1}=e_{3}=(1-q) \varepsilon_{s}$,

where $q$ is an anisotropy parameter with $q=0$ for isotropic materials.

By inserting Eqs. (7), (9) and (11) into Eq. (8) the following relationship is obtained

$\sigma_{i j}=\frac{E}{1+v}\left\{\varepsilon_{i j}-\epsilon_{i j}^{s}+\delta_{i j} \frac{v}{1-2 v}\left(\varepsilon_{k k}-3 \Lambda \epsilon_{s}\right)\right\}$,

where

$\varepsilon_{i j}^{s}=\Lambda\left\{\delta_{i j}(1-q)+3 \delta_{i 2} \delta_{j 2} q\right\} \varepsilon_{s}$.

The hydrostatic pressure at dilatational free transformation, $p_{s}$, is defined according to Eqs. (2) and (12) as follows,

$p_{s}=\frac{E}{1-2 v} \varepsilon_{s}$.

Equilibrium of stresses requires

$\sigma_{i j, j}=0$,

while body forces are assumed to be absent.

\subsection{Boundary conditions}

A large body containing a straight crack, as shown in Fig. 1, is considered. The Cartesian coordinate system 


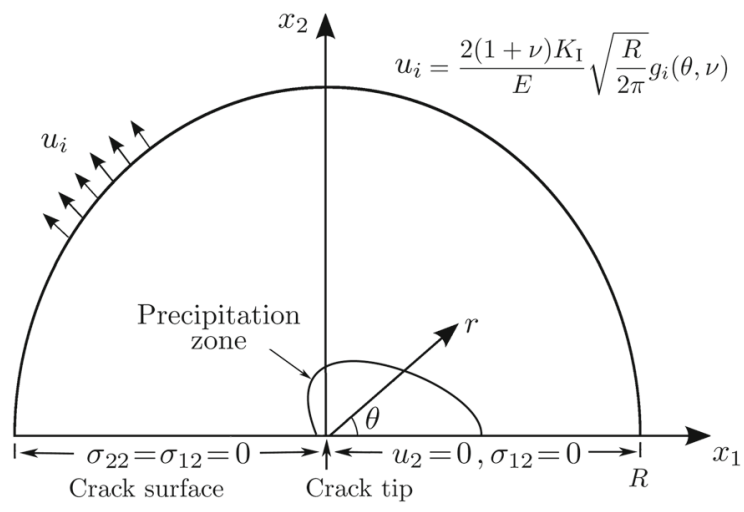

Fig. 1 Semi-infinite crack, near crack tip precipitate zone under remote mode I loading. The boundary conditions are prescribed displacements on the half circular contour, traction free crack surfaces and symmetry conditions in the crack plane ahead of the crack tip

$x_{i}$ is attached to the crack tip. The crack occupies the region $x_{1} \leq 0$ and $x_{2}=0$, and it is assumed that the precipitate only occupies a small region close to the crack tip.

Using Eqs. (6), (12) and (15) the stress and strain distributions are obtained. Initially, the body is stress free and free from precipitate. The boundary conditions are formulated as a boundary layer of given displacements between the near tip region and remote constraints. The displacements are imposed at the distance $R$ from the crack tip, see Fig. 1. The radius $R$ is chosen to be around 10 times the largest linear extent of the precipitate, $r_{h}$. A polar coordinate system with $r=\sqrt{x_{1}^{2}+x_{2}^{2}}$ and $\theta=\arctan \left(x_{2} / x_{1}\right)$ is attached to the crack tip. The remote load is assumed to be an opening mode, e.g. mode I loading. Because of the symmetry, only the upper half of the body is modelled. The imposed displacements are given by

$u_{i}=\frac{2(1+v) K_{\mathrm{I}}}{E} \sqrt{\frac{R}{2 \pi}} g_{i}(\theta, v), \quad$ for $\quad 0 \leq \theta \leq \pi$,

where $K_{\mathrm{I}}$ is the mode I stress intensity factor and $g_{i}$ are known angular functions, cf. Broberg (1999).

The remaining boundary conditions are traction free crack surfaces

$\sigma_{22}=\sigma_{12}=0 \quad$ On $-R<x_{1}<0$ and $x_{2}=0$, and

$\sigma_{12}=0$ and $u_{2}=0$ On $0 \leq x_{1}<R$ and $x_{2}=0$,

The stress driven diffusion of the mobile constituent as given by the Einstein-Smoluchowski equation (1) is solved in Sect. 2.3. The one-to-one relation (5) between critical concentration and critical stress is derived using the boundary conditions of vanishing stress and small ambient concentration at large distances from the crack tip. This eliminates the coupling between the mechanical and diffusion processes to the explicitly solvable Eq. (1) leaving only the deduced critical stress given by Eq. (5).

\subsection{Equations on non-dimensional form}

To provide insight and to facilitate proper representation of the relations between the physical quantities, the governing equations are given on a non-dimensional form. As the stress at the crack tip influenced by both formation of of precipitates and remote load, the stresses scaled with critical stress instead of remote stress.

The following non-dimensional variables are introduced

$$
\begin{aligned}
& \hat{x}_{i}=\left(\frac{\sigma_{c}}{K_{\mathrm{I}}}\right)^{2} x_{i}, \quad \hat{r}=\left(\frac{\sigma_{c}}{K_{\mathrm{I}}}\right)^{2} r, \quad \hat{r}_{h}=\left(\frac{\sigma_{c}}{K_{\mathrm{I}}}\right)^{2} r_{h}, \\
& \hat{R}=\left(\frac{\sigma_{c}}{K_{\mathrm{I}}}\right)^{2} R, \quad \hat{\sigma}_{i j}=\frac{\sigma_{i j}}{\sigma_{c}}, \quad \hat{p}_{s}=\frac{p_{s}}{\sigma_{c}}, \\
& \hat{\varepsilon}_{i j}=\frac{E}{\sigma_{c}} \varepsilon_{i j}, \quad \hat{\varepsilon}_{i j}^{s}=\frac{E}{\sigma_{c}} \varepsilon_{i j}^{s}, \quad \hat{u}_{i}=\frac{E \sigma_{c}}{K_{\mathrm{I}}^{2}} u_{i} .
\end{aligned}
$$

Thus, on non-dimensional form Eqs. (6) and (12) become

$\hat{\varepsilon}_{i j}=\frac{1}{2}\left(\frac{\partial \hat{u}_{i}}{\partial \hat{x}_{j}}+\frac{\partial \hat{u}_{j}}{\partial \hat{x}_{i}}\right)$,

and

$\hat{\sigma}_{i j}=\frac{1}{1+v}\left\{\hat{\varepsilon}_{i j}-\hat{\epsilon}_{i j}^{s}+\delta_{i j} \frac{v}{1-2 v}\left(\hat{\varepsilon}_{k k}-\hat{\epsilon}_{k k}^{s}\right)\right\}$,

where

$\hat{\epsilon}_{i j}^{s}=\tilde{\Lambda}(1-2 v)\left\{\delta_{i j}(1-q)+3 \delta_{i 1} \delta_{j 1} q\right\} \hat{p}_{s}$,

and

$\hat{\epsilon}_{k k}^{s}=3 \tilde{\Lambda}(1-2 v) \hat{p}_{s}$. 
The non-dimensional parameter $\tilde{\Lambda}$ is

$\tilde{\Lambda}=\left\{\begin{array}{ll}1 & \text { if } \tilde{\sigma}_{h} \geq 1 \\ 0 & \text { if } \tilde{\sigma}_{h}<1\end{array}\right.$,

where $\tilde{\sigma}_{h}=\left(\sigma_{h}\right)_{\max } / \sigma_{c}$, in which the $\left(\sigma_{h}\right)_{\max }$ is defined according to Eq. (10). Finally, equilibrium from Eq. (15) become

$\frac{\partial \hat{\sigma}_{i j}}{\partial \hat{x}_{j}}=0$.

The non-dimensional boundary conditions are, traction free crack surfaces giving

$\hat{\sigma}_{22}=\hat{\sigma}_{12}=0$ On $-\hat{R}<\hat{x}_{1}<0$ and $\hat{x}_{2}=0$,

and the symmetry conditions along the crack plane, cf. Sect. 2,

$\hat{\sigma}_{12}=0$ and $\hat{u}_{2}=0$ On $0 \leq \hat{x}_{1}<\hat{R}$ and $\hat{x}_{2}=0$,

and prescribed displacements

$\hat{u}_{i}=(1+v) \sqrt{\frac{2 \hat{R}}{\pi}} g_{i}(\theta, v), \quad$ On $\hat{r}=\hat{R}$ and $0 \leq \theta \leq \pi$

The non-dimensional form of the governing equations Eqs. (20) to (25) with the boundary conditions Eqs. (26) to (28) shows that the parameters $\hat{p}_{s}, q$ and $v$ are the only free parameters. The equations are solved for a variation of the ratio of dilatation free pressure versus critical stress, $\hat{p}_{s}$, for isotropic and transversely isotropic materials. The variation of Poisson's ratio $v$ is normally in a small range and relatively insignificant for the change of the solution. It is therefor, is kept the same for all calculations in the present study.

\section{Numerical procedure}

An FE model is utilised to solve the boundary value problem as stated in Sect. 2.4. The commercial FE-code Abaqus, cf. Hibbitt et al. (2003), together with a user materials subroutine are as part of the implementation. The stress field is computed according to Eqs. (20), (21) and (25) with boundary conditions Eqs. (26) and (28). In the present study, Poisson's ratio is put to $v=0.34$, which is a measured value for zirconium and is also representative for many other metals such as titanium, copper, aluminium and magnesium, see Francois et al. (2012) and Hall (1970). The anisotropy factor is $q=0$ or $q=0.16$ alternatively. The latter choice is the anisotropy of a zirconium and titanium hydrides at room temperature, cf. Singh et al. (2007) and Tal-Gutelmacher and Eliezer (2004). The choice gives a ratio of the principal expansion strains of $e_{1} / e_{2}=0.634$.

The problem is symmetric across $x_{2}=0$, therefore, only the upper half of the body is computed for the convenience and efficiency. For the transversely isotropic case the larger expansion $e_{2}$ is chosen perpendicular to the crack plane $x_{2}=0$, which is the natural orientation of growing precipitates. The mesh consists of 2175 four node isoparametric fully integrated plane strain elements. To resolve the details of the crack tip stress distribution smaller, denser elements are used around the crack tip. The size of the smallest element near the crack tip is around $0.001 \hat{R}$.

The incremental numerical scheme starts with a first step with no precipitate. The obtained maximum hydrostatic stress is chosen as a critical stress. In the second step, the element at the maximum hydrostatic stress is given the expansion strain $\hat{\epsilon}_{s}$. An adjusted stress and strain distribution is then calculated. Thus, the new element, which has obtained the largest stress is now adopted as the critical stress and the second step is repeated by adding new precipitate incrementally. The procedure of the second step is then repeated until the precipitate has reached a linear extent of around $0.1 \hat{R}$ for the isotropic cases and around $0.14 \hat{R}$ for the anisotropic cases. During the entire process, the non-dimensional variables are maintained, meaning that when the critical stress is changed, the dilatation free pressure is upgraded so that the selected nondimensional pressure $\hat{p}_{s}$ remains constant throughout the calculations. For values of $\hat{p}_{s}$ exceeding a critical value, the calculations diverge before the size requirement is reached. A theoretical explanation and discussion of this behaviour are provided in the "Appendix".

\section{Results and discussion}

First in this section, some numerical aspects are investigated and discussed. This is followed by a presentation of the resulting stress fields from the FE calculations. The results are compared with an analytically estimated largest expansion that lead to stable precipitates. The 
remote stress field and the stress field in the vicinity of the crack tip reveals a crack tip shielding that is discussed later in this section.

\subsection{Numerical considerations}

The problem has only one relevant physical length scale, hence the solutions are self-similar in the sense that they are identical and independent of remote load when observed in the available length scale, i.e., $\left(K_{\mathrm{I}} / \sigma_{c}\right)^{2}$. During the calculation, the precipitate is growing from including only a single element to typically including 300 elements at the end of the calculation. At the end, the linear extent of the precipitate is $r_{h} \approx 0.1 R$ for isotropic cases and $0.14 R$ for anisotropic case. At the end of the calculation, it is necessary that the number of elements representing the precipitate is sufficient, and that the interaction with the boundary at $r=R$ is still reasonably small.

The calculations are performed for five different values of $\hat{p}_{s}=0,1,1.5,2$ and 2.5 that are well below the critical value. As is readily seen in Eq. (C-4) in the "Appendix", the stress $\hat{p}_{s}$ is limited to values less than a critical value, which is determined to be around 36 for the isotropic material and around 6 for the anisotropic material. For a $\hat{p}_{s}$ equal or larger than the critical value the precipitates, once it is initiated, it will continue to grow to unlimited sizes even in the absence of a remote load. The only effect induced by the precipitate on mechanical state is the material expansion. Therefore, the non-expanding case $\hat{p}_{s}=0$ is identical to a case with no precipitate present.

\subsection{Precipitate shapes and sizes}

The distribution of the hydrostatic stress in the crack tip region before the precipitate is present, i.e. for $\hat{p}_{s}=0$ is shown in Fig. 2.

For isotropic materials, the expansion causes rather small changes of the shape of the precipitate for all examined values of $\hat{p}_{s}$. This is observed in Fig. 3a for $\hat{p}_{s}=2.5$, which shows the growing precipitate. During the growth, the value of $\sigma_{c}$ changes, which increases the length scale $\left(K_{\mathrm{I}} / \sigma_{c}\right)^{2}$ so that the numerically calculated precipitate covers an increasing number of elements and an increasing part of the total mesh. Still, as the length scale $\left(K_{\mathrm{I}} / \sigma_{c}\right)^{2}$ serves as the only physical

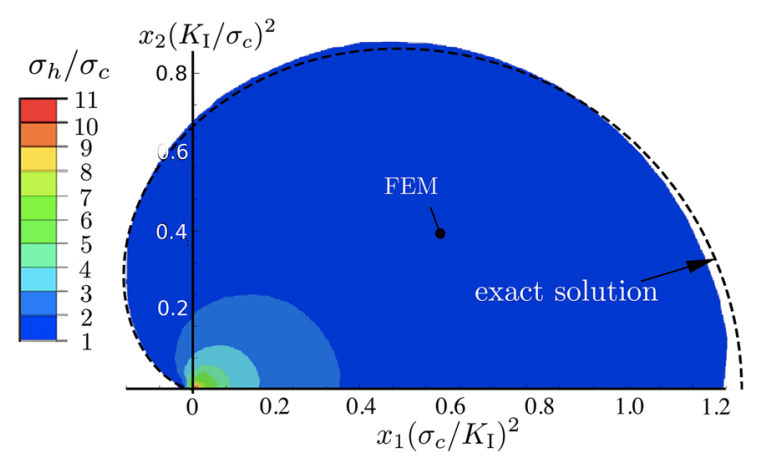

Fig. 2 The contour plot of the distribution of the hydrostatic stress for isotropic and anisotropic cases with zero expansion $\hat{p}_{s}=p_{s} / \sigma_{c}=0$. The dashed curve is the exact result as given by Eq. (16). The mesh size is $\hat{R}=R\left(\sigma_{c} / K_{\mathrm{I}}\right)^{2} \approx 12$

length reference, all curves are numerically different results of the same case. Figure $3 \mathrm{~b}$ shows the same cases as in Fig. 3a with the coordinates normalised with $\left(K_{\mathrm{I}} / \sigma_{c}\right)^{2}$. The differences in details reflect that the result is given for different precipitate sizes as compared with the mesh, i.e. for different $r_{h} / R$. A notable observation is that the shape is distorted in the beginning by rather few elements covering the precipitate and, at the end, by relatively large elements that cover the foremost part of the precipitate. The reason for this is that the elements are increasing in size at larger distances from the crack tip. Here, an average shape is believed to be more precise.

Figure 4 show the shapes of the precipitates for the isotropic and anisotropic cases for different values of $\hat{p}_{s}$. As observed for the isotropic cases in Fig. 4a, there is a slightly increased height in the foremost part with increasing dilatational free pressure $\hat{p}_{s}$. The overall influence of the expansion on the precipitate shape is rather small. It is shown that the shape of the precipitate in the isotropic case is more circular, while a wedge shaped precipitate is obtained in the anisotropic case. This is not totally surprising considering that a precipitate with the large expansion in the $x_{2}$ direction gives a stress concentration ahead of the precipitate and as a consequence, it will give a greater flux of constituent to the area. The obtained shape for the anisotropic case is consistent with earlier experimental and theoretical studies, e.g., Cann and Sexton (1980), Metzger and Sauve (1996) and Kerr (2009).

The amount of dilatation free pressure $\hat{p}_{s}$ influences the normalised length of the precipitate in the crack plane, $\hat{r}_{h}$, as is shown in Fig. 5. As it can be seen, 


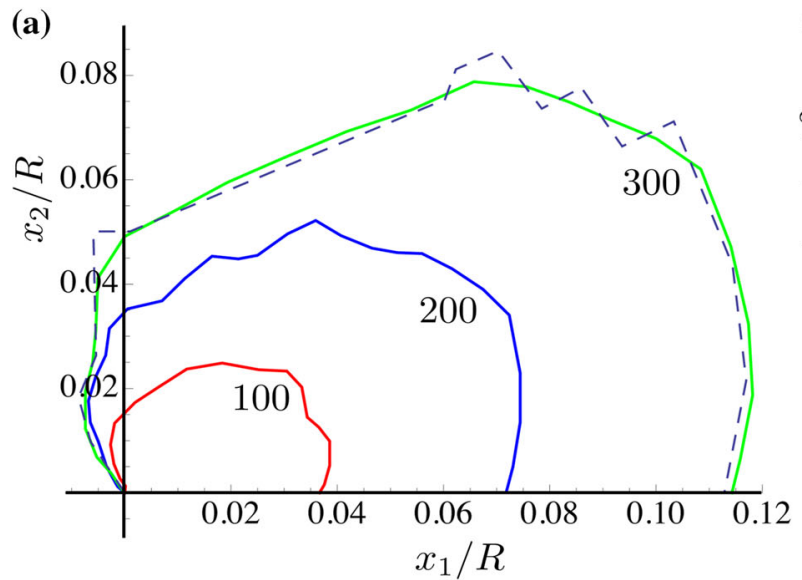

Fig. 3 The growing shape of the precipitate for the case of $\hat{p}_{s}=2.5$ for different $r_{h} / R$ in coordinates scaled with a the mesh size $R$ and $\mathbf{b}$ the length unit $\left(K_{\mathrm{I}} / \sigma_{c}\right)^{2}$. The labels 100, 200

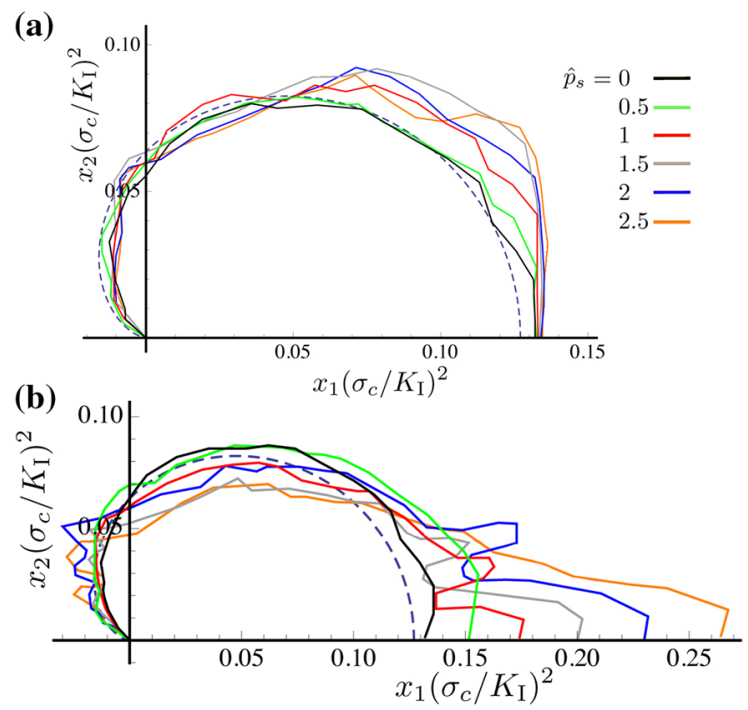

Fig. 4 The shape of precipitates for a the isotropic $(q=0)$ and $\mathbf{b}$ the anisotropic case with anisotropy factor $q=0.16$, for different dilatation free pressure $\hat{p}_{s}$. The dashed curve is the exact result for $\hat{p}_{s}=0$

as long as the precipitate is small compared with the mesh, there are noticeable fluctuations of $\hat{r}_{h}$. This is an expected consequence of the coarseness of the mesh when the precipitate covers too few elements. As the size of the precipitate increases to, e.g., $r_{h} \geq 0.015 R$, the values $\hat{r}_{h}$ are rather stable in the isotropic case and $r_{h} \geq 0.03 R$ for the anisotropic case. This shows that the precipitate size is greatly influenced by the mesh,

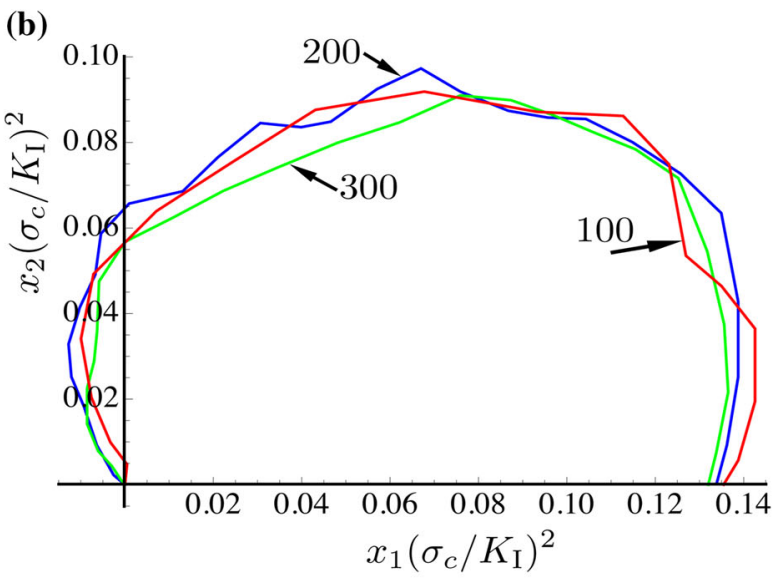

and 300 refer to the number of elements covered by the precipitate. The dashed curve shows the external contour of the elements when 300 elements cover the precipitate

thus this stage I is called mesh dependent stage. At stage I, the $r_{h}$ is as a minimum 15 times larger than the smallest elements, also the mesh then encompass around 20 to 50 elements. After this, in stage II, the fluctuations in $\hat{r}_{h}$ decrease and become stable at the end of stage II. There is also a third stage, in which the result is affected by the finite dimensions of the mesh to precipitate ratio. The effect is rather small, and not visible until the length of the precipitate exceeds around a third of the mesh radius $R$. It is therefore not visible in the range of Fig. 5. It seems that the selected mesh fulfils the requirements of mesh independence in the region of $0.015<r_{h} / R \leq 0.1$ for the isotropic case and in $0.03<r_{h} / R \leq 0.13$ for the anisotropic case. Thus, the size of the precipitate can be extracted from the FEM result in stage II.

As stated in Sect. 4.1, the problem only involves a single relevant physical length that scale all characteristic lengths. This is observed in Fig. 5 where the nondimensional linear extent, $\hat{r}_{h}$, of the precipitate in the crack plane essentially does not change during growth in stage II. A small decay with less than $2 \%$ of $\hat{r}_{h}$ for $\hat{p}_{s}=0.5$ and around $5 \%$ for $\hat{p}_{s}=2.5$, i.e., the top curve in Fig. 5a. In the isotropic case, cf. Figure 5b, the changes are almost insignificant. This confirms that the problem is self-similar and that further refinement of the mesh affects neither to the shape nor to the size of the precipitate. Fig. 5 also indicates that $\hat{r}_{h}$ becomes less stable and some fluctuations occur for larger values of $\hat{p}_{s}$, meaning that the maximum amplitude of 

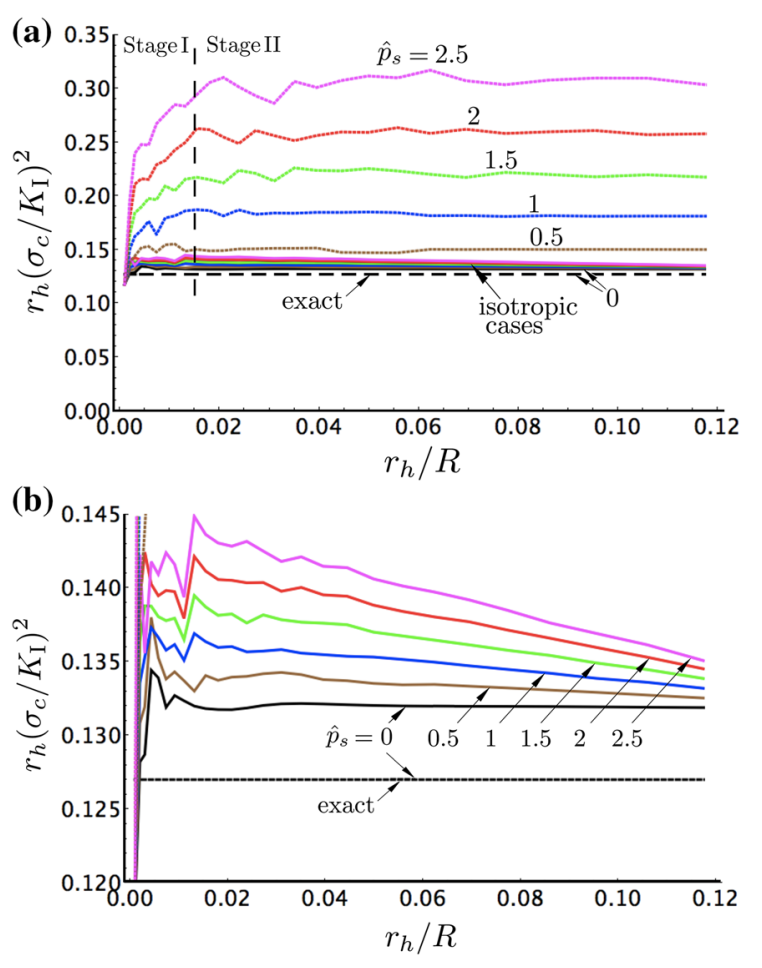

Fig. $5 \hat{r}_{h}=r_{h}\left(\sigma_{c} / K_{\mathrm{I}}\right)^{2}$ versus $r_{h} / R$ for a isotropic and anisotropic (dotted) cases. b A magnification showing the details of the isotropic case. The exact result for $\hat{p}_{s}=0$ is included

fluctuation is $5 \%$ normalised size of precipitate $\hat{r}_{h}$ for anisotropic case. In a whole, the different values of $\hat{p}_{S}$ has great effect on the normalised size of precipitate in anisotropic case, c.f. Metzger and Sauve (1996). The details of estimation of the linear extent of the precipitate is given in the "Appendix", where it is shown that above a certain limit of $\hat{p}_{s}$ the precipitate size becomes unbounded. More precisely, when the expansion approaches a certain level, the influence of the required remote load approaches zero. This means that the precipitate, once it is initiated, will continue to grow even after the remote load is removed, cf. "Appendix" (C-4).

\subsection{Stresses ahead of the crack}

As expected, the formation of precipitate initiates at the crack tip, where the hydrostatic stress assumes its maximum value, see Fig. 2.

At the boundary of the precipitate, according to Eq. (21), there must be a jump in $\hat{\sigma}_{h}, \hat{\sigma}_{22}$ and $\hat{\sigma}_{33}$ across the interface between the precipitate and the original material. This jumps may be calculated using Eqs. (21), (23) and (24). The conditions are that the stress $\hat{\sigma}_{11}$, the strain $\hat{\epsilon}_{22}$, and $\hat{\epsilon}_{33}$ remain continuous across the interface. The following is obtained:

$$
\begin{aligned}
\Delta \hat{\sigma}_{h} & =(2+q) \frac{1-2 v}{3(1-v)} \hat{p}_{s}, \\
\Delta \hat{\sigma}_{22} & =\left(1+\frac{2-v}{1+v} q\right) \frac{1-2 v}{1-v} \hat{p}_{s}, \\
\Delta \hat{\sigma}_{33} & =\left(1-\frac{1-2 v}{1+v} q\right) \frac{1-2 v}{1-v} \hat{p}_{s},
\end{aligned}
$$

for $\hat{p}_{s}=2.5$ and $v=0.34$ give $\Delta \hat{\sigma}_{h}=0.81, \Delta \hat{\sigma}_{22}=$ 1.21 and $\Delta \hat{\sigma}_{33}=1.21$ for $q=0$ in the isotropic case, and $\Delta \hat{\sigma}_{h}=0.87, \Delta \hat{\sigma}_{22}=1.45$ and $\Delta \hat{\sigma}_{33}=1.17$ for $q=0.16$ in the anisotropic case. The numerical results of jumps from finite element calculation can be evaluated from Figs. 6, 7, and 8.

In Fig. 6, the hydrostatic stresses $\hat{\sigma}_{h}=\sigma_{h} / \sigma_{c}$ in the crack plane versus the distance from the crack tip $x_{1} / r_{h}$ for different values of $\hat{p}_{s}$ are shown. As expected the expansion causes the stresses inside the precipitate to decay for both isotropic and anisotropic cases. A small fluctuation occurs closely around the boundary of the precipitate in the anisotropic case. With selected value, e.g. $\hat{p}_{s}=2.5$ one obtains a theoretical jump in the hydrostatic stress of $\Delta \hat{\sigma}_{h}=0.81$, according to Eq. (29), which is fairly close to the obtained result $\Delta_{\text {FEM }} \hat{\sigma}_{h} \approx 0.801$. Similarly, for the same value of $\hat{p}_{s}$ and $q=0.16$, the theoretical jump in hydrostatic stress in the anisotropic case is $\Delta \hat{\sigma}_{h}=0.87$, and $\Delta_{\mathrm{FEM}} \hat{\sigma}_{h} \approx$ 0.86 .

One may notice that, the hydrostatic stresses in the crack plane ahead of the precipitates are seemingly independent of $\hat{p}_{s}$ in the isotropic case, whereas in the anisotropic case the expansion of precipitate significantly increases the hydrostatic stress immediately ahead of the precipitate. A consequence would be an enhanced growth rate of the precipitate in the anisotropic case, while the gradient of the hydrostatic stress increases with the expansion. It is not fully understood whether this is a numerical effect or a reality. The present assumption is that it is caused by the rapid changes at the adjacent precipitate interface.

Figure 7 shows the stress normal to the crack plane $\hat{\sigma}_{22}=\sigma_{22} / \sigma_{c}$ versus the distance from the crack tip $x_{1} / r_{h}$ for different values of $\hat{p}_{s}$. The stress is decreasing with increasing distance from the crack tip inside the precipitate. For the anisotropic case the stresses 

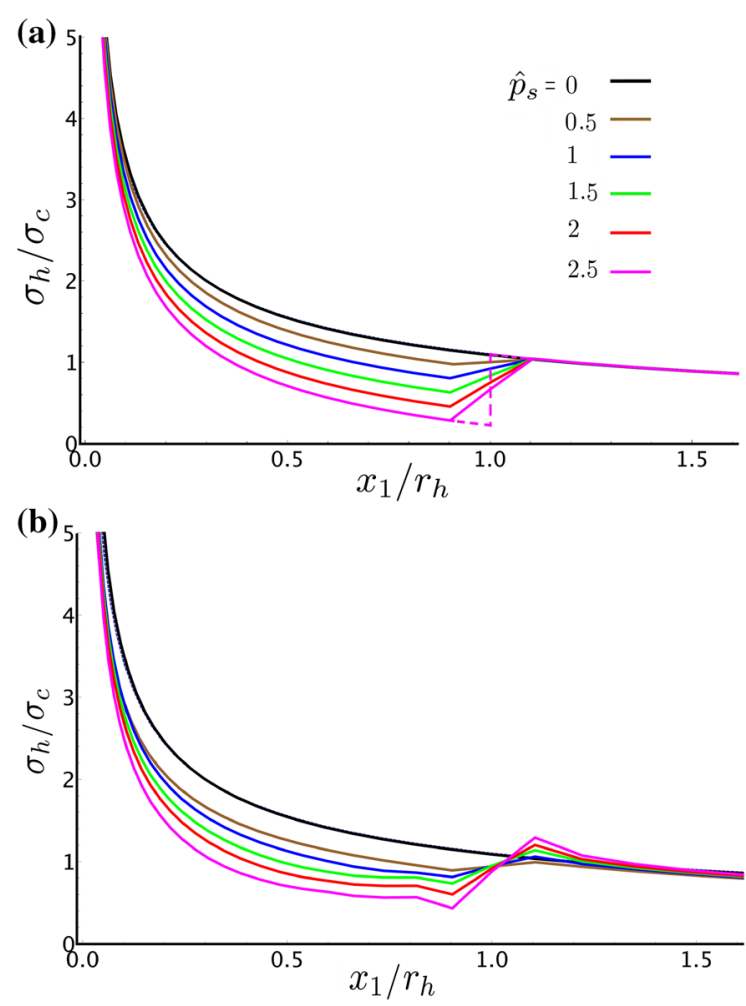

Fig. 6 The normalised hydrostatic stress $\hat{\sigma}_{h}=\sigma_{h} / \sigma_{c}$ in the crack plane versus scaled distance $x_{1} / r_{h}$ ahead of crack tip for different values of $p_{s} / \sigma_{c}=0,0.5,1,1.5,2,2.5$, for a isotropic and $\mathbf{b}$ anisotropic (transversely isotropic) cases. The dashed line is manually extended FEM results for $\hat{p}_{s}=2.5$ to show the jump in hydrostatic stress that cannot be accurately resolved in the FEM-calculations. The analytical result for $\hat{p}_{s}=0$ is included but coincides with the numerical result

outside precipitates are not only lower than for the isotropic case, but there is also a noticeable fluctuation of the stresses inside the precipitate. This starts at around $x_{1} \approx 0.8 r_{h}$ and larger distances. Nevertheless, this behaviour does not continue outside of precipitate, and at some distance outside of precipitate all stresses closely follow the same curve as that without a precipitate, see Fig. 7.

The stress $\sigma_{11}$ is continuous across the precipitate interface. This is also clearly observed for the isotropic case in Fig. 8a. The stress $\sigma_{11}$ for the isotropic case is slightly decreased by the expansion both in the precipitate and outside of the precipitate. For the anisotropic case, there is a rapid change of $\hat{\sigma}_{11}$ in the vicinity of the precipitate interface that should not be confused with a stress discontinuity. As is indicated, the rapid changes of the stress in the vicinity of the precipitate interface cover, in this case, only a few finite elements.
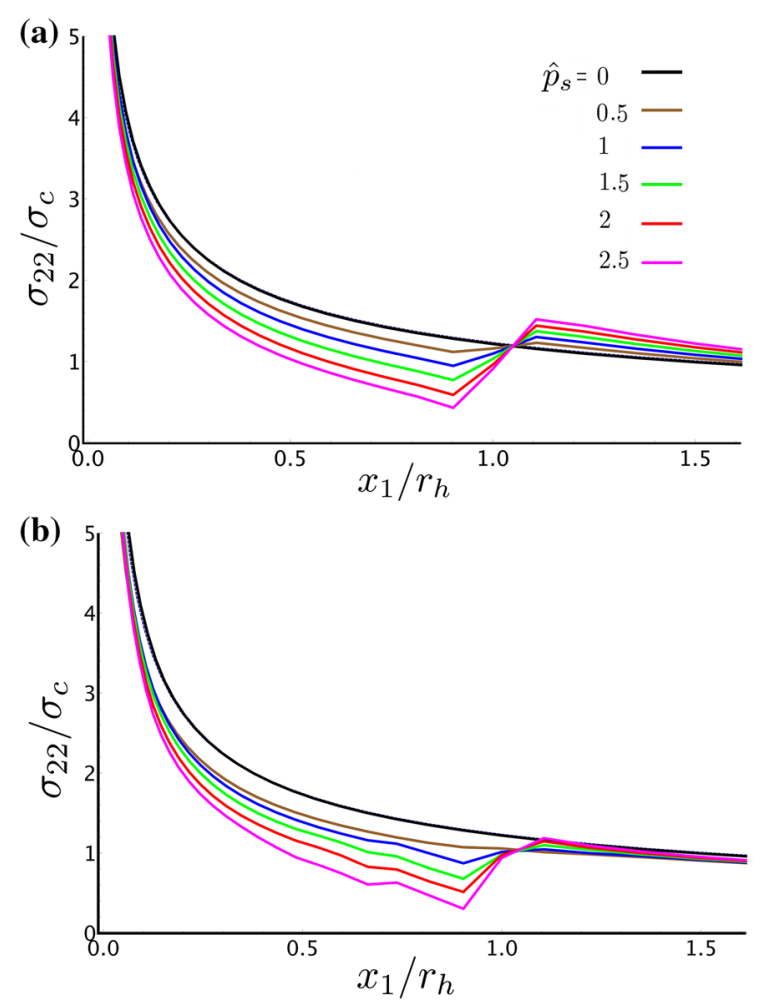

Fig. 7 The normalised stress $\hat{\sigma}_{22}=\sigma_{22} / \sigma_{c}$ in the $x_{2}$ direction in the crack plane versus scaled distance $x_{1} / r_{h}$ ahead of crack tip for different values of $\hat{p}_{s}=p_{s} / \sigma_{c}=0,1,1.5,2,2.5$, for $\mathbf{a}$ isotropic and $\mathbf{b}$ anisotropic cases

\subsection{Crack tip shielding}

The expanded phase decreases the stresses ahead of the crack tip, according to Figs. 6 and 7. The stress in the closest vicinity of the crack tip cannot be resolved from the aforementioned figures. To make the comparison between the materials with different $\hat{p}_{s}$, the stress intensity factor of the crack tip stress field is calculated using the relation

$K_{t i p}=\lim _{x_{1} \rightarrow 0} \sigma_{22} \sqrt{2 \pi x_{1}}$,

where the stress $\sigma_{22}$ is the stress across in the crack plane inside the precipitate. Figure 9 shows the relative stress intensity factor normalised with respect to the remote stress intensity factor versus the dilatational free swelling pressure $\hat{p}_{s}$. The remote stress intensity factor is defined as $K_{\text {rem }}=K_{\mathrm{I}}$ via Eq. (16). As observed, the precipitate has a mild shielding effect on the crack tip stress intensity factor. For the studied cases, the shield- 

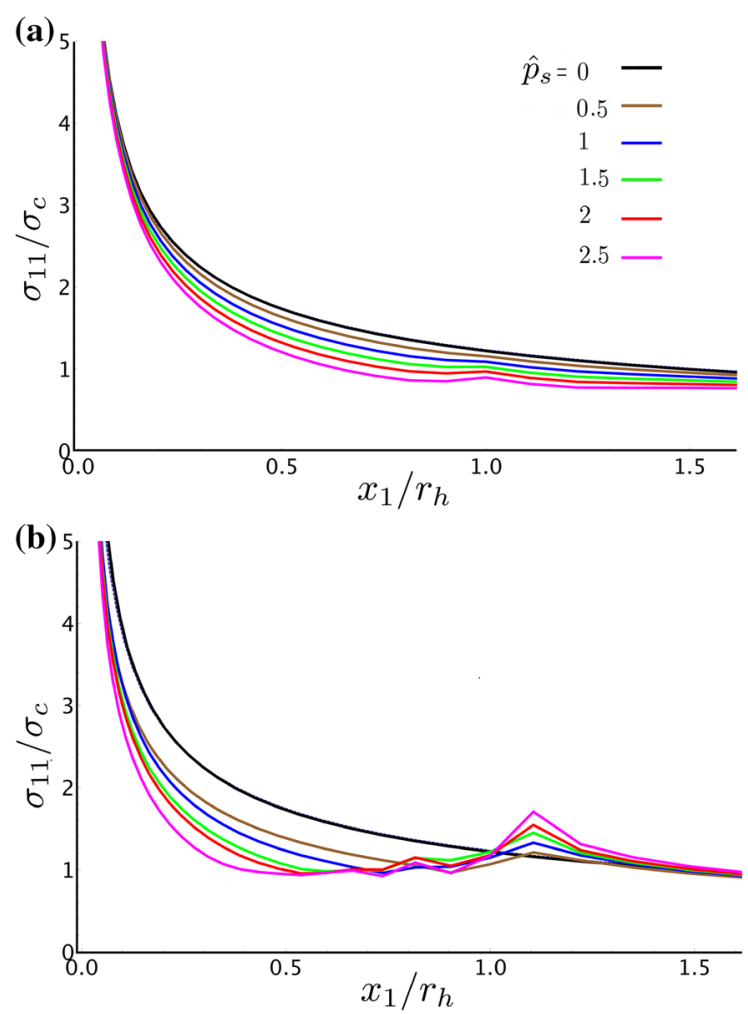

Fig. 8 The normalised stress $\hat{\sigma}_{11}=\sigma_{11} / \sigma_{c}$ in the crack plane versus scaled distance $x_{1} / r_{h}$ ahead of crack tip for different values of $p_{s} / \sigma_{c}=0,1,1.5,2,2.5$, for $\mathbf{a}$ isotropic and $\mathbf{b}$ anisotropic cases

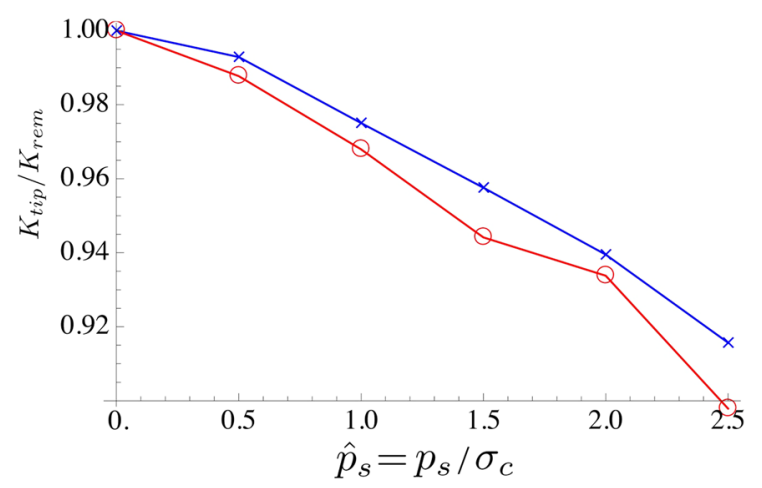

Fig. 9 The relative stress intensity factor for isotropic (blue) and anisotropic (red) cases versus the dilatation free pressure $\hat{p}_{s}=p_{s} / \sigma_{c}$

ing is increasing with increasing $\hat{p}_{s}$. The effect is rather small and around $10 \%$ for $\hat{p}_{s}=2.5$. The shielding of the anisotropic material is slightly larger, which is expected since the expansion is larger across the crack plane in this case.
Table 1 Mechanical and chemical properties of zirconium and zirconium hydride system Cann and Sexton (1980), Leitch and Puls (1992) and Varias and Feng (2004)

\begin{tabular}{ll}
\hline Modulus of elasticity, $E$ & $90 \mathrm{GPa}$ \\
Linear expansion strain, $\epsilon_{s}$ & 0.057 \\
Molar volume of H diss. in $\mathrm{Zr}, V$ & $16.7 \times 10^{-7} \mathrm{~m}^{3} / \mathrm{mol}$ \\
Universal gas constant, $R$ & $8.31 \mathrm{~J} / \mathrm{K} \mathrm{mol}$ \\
Room temperature, $T$ & $293 \mathrm{~K}$ \\
Anisotropy parameter, $q$ & 0.16 \\
Calculated critical $\hat{p}_{s}$, & 3 \\
Calc. crit. ambient conc. ratio, $\frac{C_{o}}{C_{c}}$ & 0.328 \\
\hline
\end{tabular}

\section{Application to zirconium and zirconium hydride}

Zirconium is an important construction material used in nuclear power production. The advantage of zirconium and its alloys is a low neutron absorption cross section, resistance to radiation damage and corrosion Raj et al. (2008).

Zirconium has a fairly large expansion due to presence of hydrogen in solid solution and would normally cause expansion of the zirconium. Not as much as at a phase transformation from zirconium to zirconium hydride but still significant. However, due to the depletion of hydrogen in a region surrounding the hydride the expansion of the zirconium decreases and may be ignored, cf. Puls (2012) and Louthan Jr (2008)

The following data, in Table 1, can be used for estimation of the critical ambient concentration of hydrogen that leads to self-growing of hydrides.

Utilising Eq. (5) one obtains the critical ambient stress

$C_{o}=C_{c} \exp \left(\frac{-E \epsilon_{s} V}{\hat{p}_{s}(1-2 v) R T}\right)$.

By using the material data in Table 1 and Eq. (31) one obtains $C_{o}=0.328 C_{c}$. The meaning of this is that the zirconium hydride will grow spontaneously, i.e. without requiring support from any remote load at an ambient concentration that is around $16 \%$ of what is expected to be the terminal solid solubility. It is the residual stress caused by the expanding hydride that supports the drag on the hydrogen towards the crack tip. Therefore, it is required that a hydride must already be established at the crack tip.

Further, using material data from Table 1, it is found that the ambient hydrogen concentration that is covered 


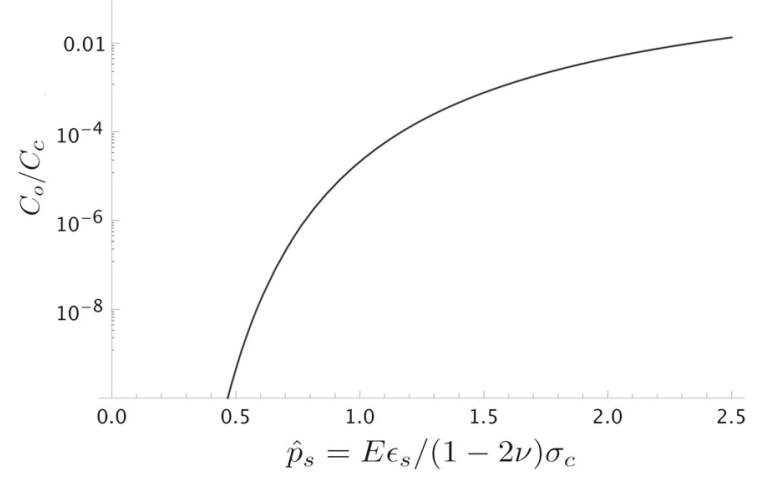

Fig. 10 Ratio of ambient concentration $C_{o}$ and terminal solid solubility of hydrogen in zirconium $C_{c}$ versus normalised expansion pressure $\hat{p}_{s}$ Eq. (31)

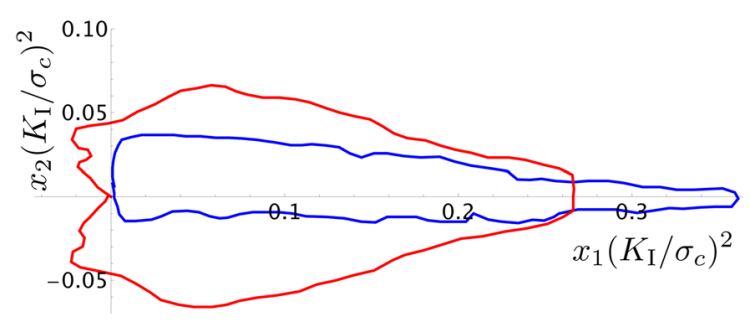

Fig. 11 Comparison of an observed crack tip hydride formed in zirconium (blue) (cf. Kerr 2009; Metzger and Sauve 1996) with the result for the anisotropic case and $\hat{p}_{s}=2.5$ (red)

by the range $0 \leq \hat{p}_{S} \leq 2.5$ is $0 \leq C_{o} / C_{c} \leq 0.0135$. The result is shown in Fig. 10.

A typical image of a crack tip hydride in zirconium is found in Cann and Sexton (1980), Metzger and Sauve (1996) and Kerr (2009). This provides an opportunity to compare the observed shape with the shapes obtained in the present study. In Fig. 11 the comparison is made of an observed crack tip hydride formed in zirconium with the result for the anisotropic case and $\hat{p}_{s}=2.5$. The size cannot be related since the crack tip load $K_{\mathrm{I}}$ causing the real hydride is unknown. The size of the real hydride has therefore been adjusted to a size suitable for comparing their shapes. The contour is digitised from a scanning electron microscope image in Kerr (2009) and Metzger and Sauve (1996), in Fig. 11. Considering the increasing flattening of the precipitate with increasing $\hat{p}_{s}$ a possible reason for the mismatching results could be that the real hydride is produced at a lower ambient concentration than corresponding to $\hat{p}_{s}=2.5$.

\section{Conclusions}

The growth of expanding precipitates at the tip of a stationary crack are studied using both analytical and numerical methods. The hydrostatic stress in crack tip regions is known to drag the constituent in solid solutions to the crack tip region. When the concentration reaches the solid solubility limit precipitation initiates and grows around the crack tip. By direct integration of Einstein-Smoluchowski equation, it is shown that a theoretical concentration criterium along the fringe can be replaced with a criterium for critical hydrostatic stress, $\sigma_{c}=\frac{R T}{V} \ln \frac{C_{c}}{C_{o}}$, where the ratio of the solid solubility limit $C_{c}$ versus the remote ambient concentration $C_{o}$, controls the growth of the precipitate. The implication is that high ambient concentration, $C_{o}$ decrease critical stress and increase the chance for self-growth. Also the normalised pressure, $\hat{p}_{s}=p_{s} / \sigma_{c}$, at dilatation free transformation becomes large.

On the non-dimensional form of equations, the free parameters are the expansion strain, the degree of anisotropy and Poisson's ratio. The influence of Poisson's ratio is not examined. The problem has only a single length scale, e.g., $\left(K_{\mathrm{I}} / \sigma_{c}\right)^{2}$ and as a consequence the precipitate grows under self-similar conditions. It is shown that, based on an assumption of self-similar growth, one can obtain a closed form solution of the precipitate size, cf. "Appendix" (C-4). There it is shown that the precipitate will grow even if the remote load is removed for large transformation expansions.

The selected numerical method is an incrementally updated FE calculation where every increment reforms the shape of the precipitate. A comparison with an observed zirconium hydride shape shows that the shape has a clear resemblance, but seems to originate from a lower ambient concentration giving a larger $\hat{p}_{S}$ than the highest value 2.5 studied in this analysis.

Acknowledgements Financial support from The Swedish Research Council under Grant No. 2011-5561 and ÅForsk Foundation under Grant No. 18-489 are gratefully acknowledged.

Open Access This article is distributed under the terms of the Creative Commons Attribution 4.0 International License (http:// creativecommons.org/licenses/by/4.0/), which permits unrestricted use, distribution, and reproduction in any medium, provided you give appropriate credit to the original author(s) and the source, provide a link to the Creative Commons license, and indicate if changes were made. 


\section{Appendix: Estimate of the linear extent of the pre- cipitate}

Considering a precipitate immediately in front of a crack tip in the absence of remote load, the only relevant parameters are the dilatation free pressure $p_{s}$ and the anisotropy parameter $q$. Let the hydrostatic stress immediately ahead of the precipitate be

$\sigma_{h}=\xi_{o} p_{s}$

where the positive dimensionless constant $\xi_{o}$ may be a function of $q$ but independent of $p_{s}$. In the isotropic case for which the shape of the precipitate is little affected by $p_{s}$. In anisotropic cases the shape is affected by $p_{s}$ but the effect on $\xi_{o}$ caused by variation of $p_{s}$ is found to be almost insignificant. The constant $\xi_{o}$ is computed for both constrained displacements and for traction free remote boundaries. The average is taken as an estimated $\xi_{o}$. The resulting estimates for $\xi_{o}$ are, with rather small variation due to varying $p_{s}, 0.028$ and 0.161 for both the isotropic $(q=0)$ and anisotropic ( $q=0.16)$ cases respectively.

Let the extent of the precipitate in the crack plane be denoted $r_{h}$. Suppose that a remote mode I load is applied. Because of the linear behaviour of the material the hydrostatic stresses of a mode I crack may be directly superimposed. In the crack plane the hydrostatic stress becomes

$\sigma_{o}=\xi_{o} p_{s}+\frac{2(1+v)}{3} \frac{K_{\mathrm{I}}}{\sqrt{2 \pi r_{h}}}$,

see e.g. Broberg (1999). It is assumed that the precipitate extends to $x_{1}=r_{h}$ ahead of the crack tip.

To form a precipitate the hydrostatic stress must reach the critical stress as is given by Eq. (5). When the hydrostatic stress immediately outside the precipitate reaches the required critical stress $\sigma_{o}=\sigma_{c}$ according to the condition (24). The non-dimensional form of (C-2) becomes,

$1=\xi_{o} \hat{p}_{s}+\frac{2(1+v)}{3} \frac{1}{\sqrt{2 \pi \hat{r}_{h}}}$

where $\hat{r}_{h}=r_{h}\left(\sigma_{c} / K_{\mathrm{I}}\right)^{2}$. Now an approximate extent of the precipitate is obtained as,

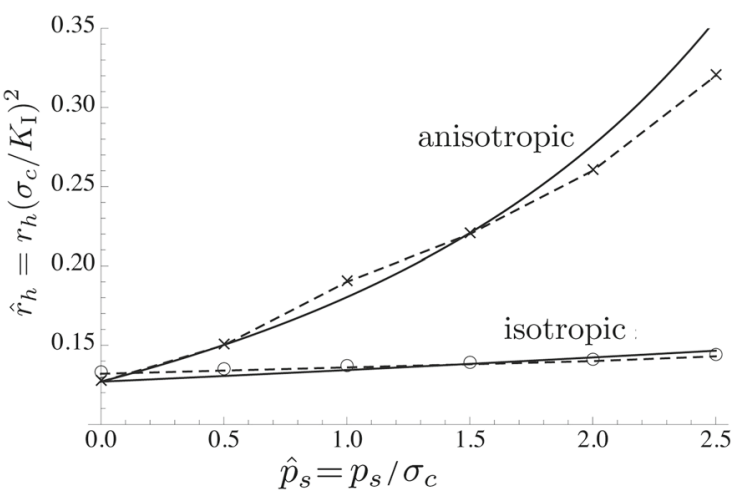

Fig. 12 Approximate result according to Eq. (C-4) (dashed) and the FEM result for the isotropic and the anisotropic materials. The extent, $\hat{r}_{h}$, of the precipitate is evaluated as in Fig. 5 for $r_{h}=0.1 R$

$\hat{r}_{h}=\frac{2(1+v)^{2}}{9 \pi} \frac{1}{\left(1-\xi_{o} \hat{p}_{S}\right)^{2}}$.

According to the result the precipitate size is determined by $\hat{p}_{s}$ and the constant $\xi_{o}$ defining the stress ahead of the precipitate.

Figure 12 shows the linear extent of the precipitate for different expansion stresses as the FEM result and the analytical prediction. It is interesting to note that $r_{h}$ is rather accurately given by Eq. (C-4). It is also noted the growth rate increases with increasing $\hat{p}_{s}$. As is readily seen in Eq. (C-4) the precipitate size becomes unbounded as $\hat{p}_{S}$ approach $1 / \xi_{o}$ which the dilatation free pressure to around $\hat{p}_{s}=36$ for the isotropic case and to around $\hat{p}_{s}=6.2$ for the anisotropic case.

\section{References}

Bertolino G, Meyer G, Perez Ipiña J (2003) Effects of hydrogen content and temperature on fracture toughness of zircaloy-4. J Nucl Mater 320(3):272-279

Broberg K (1999) Cracks and fracture. Academic Press, San Diego (ISBN: 0121341305)

Cann C, Sexton E (1980) An electron optical study of hydride precipitation and growth at crack tips in zirconium. Acta Metall 28(9):1215-1221

Carpenter G (1973) The dilatational misfit of zirconium hydrides precipitated in zirconium. J Nucl Mater 48(3):264-266

Courtney TH (2005) Mechanical behavior of materials. Waveland Press, Long Grove (ISBN-10: 1577664256)

Ellyin F, Wu J (1994) Effect of hydride precipitation on the elastoplastic stress field near a crack tip. Acta Metall Mater 42(8):2709-2717 
Farrow M, Watkins B (1965) The effect of hydrogen on the embrittlement of zirconium-base pressure tubes. J Nucl Mater 15(3):208-219

Francois D, Pineau A, Zaoui A (2012) Mechanical behaviour of materials. Springer, Dordrecht

Garverick L (1994) Corrosion in the petrochemical industry. ASM International, Materials Park (ISBN-10: 1577664256)

Hall EO (1970) Yield point phenomena in metals and alloys. Plenum Press, New York

Hibbitt, Karlsson, Sorensen (2003) ABAQUS Theory Manual (version 6.8). Hibbitt, Karlsson and Sorenson Inc., New York

Kerr M (2009) Mechanical characterization of zirconium hydrides with high energy X-ray diffraction. Ph.D. thesis, Queens University, Ontario, Canada

Leitch BW, Puls MP (1992) Finite element calculations of the accommodation energy of a misfitting precipitate in an elastic-plastic matrix. Metall Trans A 23(3):797-806

Li JCM (1978) Physical chemistry of some microstructural phenomena. Metall Trans A 9(10):1353-1380

Louthan M Jr (2008) Hydrogen embrittlement of metals: a primer for the failure analyst. J Fail Anal Prev 8(3):289-307

Lufrano J, Sofronis P (1996) Numerical analysis of the interaction of solute hydrogen atoms with the stress field of a crack. Int J Solids Struct 33(12):1709-1723

Metzger D, Sauve R (1996) A self-induced stress model for simulating hydride formation at flaws. In: Computer technology1996: Applications and methodology. PVP, vol 326, New York

Moan G, Rudling P (2002) Zirconium in the nuclear industry: thirteenth international symposium, vol 1423. ASTM, West Conshohocken, PA, USA

Puls M (2012) The effect of hydrogen and hydrides on the integrity of zirconium alloy components: delayed hydride cracking. Springer, London
Raj B, Vijayalakshmi M, Rao PV, Rao K (2008) Challenges in materials research for sustainable nuclear energy. MRS Bull 33(04):327-337

Shi SQ (1999) Diffusion-controlled hydride growth near crack tip in zirconium under temperature transients. J Nucl Mater 275(3):318-323

Singh R, Kumar N, Kishore R, Roychaudhury S, Sinha T, Kashyap B (2002) Delayed hydride cracking in $\mathrm{Zr}-2.5 \mathrm{Nb}$ pressure tube material. J Nucl Mater 304(2):189-203

Singh R, Mukherjee S, Gupta A, Banerjee S (2005) Terminal solid solubility of hydrogen in $\mathrm{Zr}$-alloy pressure tube materials. J Alloys Compd 389(1):102-112

Singh R, Ståhle P, Massih A, Shmakov A (2007) Temperature dependence of misfit strains of $\delta$-hydrides of zirconium. $\mathbf{J}$ Alloys Compd 436(1):150-154

Svoboda J, Fischer F (2012) Modelling for hydrogen diffusion in metals with traps revisited. Acta Mater 60(3):1211-1220

Tal-Gutelmacher E, Eliezer D (2004) Hydrogen-assisted degradation of titanium based alloys. Mater Trans 45(5):15941600

Turnbull A, Ferriss D, Anzai H (1996) Modelling of the hydrogen distribution at a crack tip. Mater Sci Eng A 206(1):1-13

Varias A, Feng J (2004) Simulation of hydride-induced steadystate crack growth in metals-part 1: growth near hydrogen chemical equilibrium. Comput Mech 34(5):339-356

Weatherly G (1981) The precipitation of $\gamma$-hydride plates in zirconium. Acta Metall 29(3):501-512

Publisher's Note Springer Nature remains neutral with regard to jurisdictional claims in published maps and institutional affiliations. 